\title{
Wirtschaftspolitische Positionen österreichischer Parteien im historischen Verlauf \\ Die Ausgestaltung österreichischer Parteiprogrammatiken hinsichtlich neoliberalen Gedankenguts
}

\begin{abstract}
Christian Grimm*
Zusammenfassung

Diese Arbeit analysiert die Grundsatzprogramme der einflussreichsten österreichischen Parteien (ÖVP, SPÖ, FPÖ, Grüne) vor dem Hintergrund einer historisch gewachsenen Hegemonie des Neoliberalismus. Mittels einer vergleichenden Längsschnittanalyse wird der Einfluss der neoliberalen Wende in den späten 197oern auf die Inhalte der Programmatiken identifiziert. Im Ergebnis lässt sich festhalten, dass die aktuellen Programme aller vier Parteien neoliberalen Standpunkten näherstehen als ihre analysierten Vorgängerschriften. Gleichwohl konnten Variierungen bei der Intensität der Annäherung nachgewiesen werden.
\end{abstract}

Schlagwörter: Österreichische Parteiprogrammatiken, marktliberale Prinzipien, Neoliberalismus, Wirkungsmacht ökonomischer Theorien

\section{Economic Policy Positions of Austrian Parties in Historical Course}

The Impact of Neoliberalism in Austrian Party Manifestos

\section{Abstract}

Focus of this article is to analyse manifestos from Austrian parties concerning the historical neoliberal hegemony. The central aim is to detect how far content-related changes in party manifestos were effected by the neoliberal turn in the late nineteen seventies. Therefore an empirical longitudinal analysis was conducted, in which the currently manifestos were compared with those, who were not ascribed to the neoliberal age. The results show an increased impact of neoliberal contents in all current political programmes.

Keywords: Austrian Party Manifestos, Liberal Principles, Neoliberalism, Performativity of Economics

${ }^{*}$ Christian Grimm, Johannes Kepler Universität Linz (JKU), Forschungsinstitut für die Gesamtanalyse der Wirtschaft. E-Mail: Christian.Grimm@jku.at 


\section{1. Ökonomietheorien, Neoliberalismus und politische Grundsatzprogramme}

Im Allgemeinen werden parteipolitische Grundsatzprogramme in der politikwissenschaftlichen Forschung im Vergleich zu Aktions- oder Wahlprogrammen weniger als konkrete Anleitung für aktuelle politische Tagesthemen denn als langfristige und abstrakte Leitlinienvorgabe charakterisiert, aus der sich v. a. das Selbstverständnis einer Partei ableiten lässt (Flohr 1968; Flechtheim 1973). Obgleich Grundsatzprogrammen primär nach innen gerichtete Funktionen (z. B. Integrations-, Identifikations- und Legitimationsfunktion) zugeschrieben werden, erfüllen die darin herausgearbeiteten ideologischen Grundwerte, Forderungen und Zielsetzungen auch den Zweck der Konstituierung einer parteispezifischen Identität nach außen (z. B. Werbefunktion, Profilfunktion), insbesondere im Hinblick auf eine programmatische Abgrenzung zur Konkurrenz im demokratischen Wettbewerb (Kaack 1971). Im Entstehungsprozess von Grundsatzprogrammen nehmen parteiinterne Führungspersönlichkeiten für gewöhnlich keine alleinig bestimmende Rolle ein. Entscheidender sind die etwa auf (Sonder-)Parteitagen zustande kommenden Ergebnisse, die oftmals durch ideologische Grundpositionen beeinflusst sind.

Dabei - und das ist der Ausgangspunkt dieses Beitrags - war es neben diverser politischer Ideologien auch ökonomietheoretischen Strömungen immer wieder möglich, ihr spezifisches Gedankengut als eine Art common sense auf die realpolitische Entscheidungsebene zu übertragen. So stehen z. B. nicht nur die Werke von Adam Smith (1776/2001), Karl Marx (1848) und John Maynard Keynes (1936/1964) symbolisch für die Wirkungsmacht solcher Theorien zur nachhaltigen Beeinflussung wirtschaftspolitischen Handelns, sondern auch die gegenwärtige Epoche des neoliberalen (Finanz-)Kapitalismus fußt im Kern auf einer ökonomischen Konzeption. Zentraler Ursprung dieser Bewegung war das Gründungstreffen der Mont Pèlerin Society (MPS) 1947, bei dem das Ziel, den Neoliberalismus langfristig zur dominierenden Weltanschauung aufsteigen $\mathrm{zu}$ lassen, formuliert wurde (Schmelzer 2010). Der Durchbruch auf wirtschaftspolitischer Ebene (neoliberale Wende) gelang in den angelsächsischen Industriestaaten in den späten 1970er-Jahren.

Gegenstand des vorliegenden Beitrags ist eine Auseinandersetzung mit unterschiedlichen programmatischen Positionen österreichischer Parteien vor dem Hintergrund der neoliberalen Wende in der Wirt- schaftspolitik. Hierbei wird der Frage nachgegangen, welche neuen Vorstellungen und Standpunkte infolgedessen Einzug in die Grundsatzprogrammatiken hielten und inwiefern sich hieraus Unterscheidungen $\mathrm{zu}$ früheren Programmen identifizieren lassen. $\mathrm{Zu}$ diesem Zweck wird eine empirische Längsschnittanalyse anhand von vier spezifischen Dimensionen zur Untersuchung der programmatischen Ausgestaltung hinsichtlich der Intensität ihrer Annäherung auf neoliberal besetzte Inhalte durchgeführt.

Die weitere Untersuchung ist folgendermaßen strukturiert: Zu Beginn wird ein kurzer Überblick über die Historie des neoliberalen Hegemonialprojekts und einiger seiner Leitgedanken gegeben (Kapitel 2). Danach werden mit der Auswahl des Datenmaterials, der gewählten Verfahrensweise sowie der Festlegung der Untersuchungsdimensionen die methodischen Grundlagen der Analyse thematisiert (Kapitel 3). Im Anschluss daran erfolgt die Vorstellung der Ergebnisse für die einzelnen Parteien (Kapitel 4). Der Beitrag schließt mit einer zusammenfassenden Darstellung der ermittelten programmatischen Standpunkte (Kapitel 5).

\section{Wirkungsgeschichte und Leitgedanken des Neoliberalismus}

Im nachfolgenden Kapitel wird zunächst ein Überblick über die Wirkungsgeschichte des Neoliberalismus präsentiert (2.1). Im Anschluss daran werden wesentliche Leitgedanken dieses Gedankengebäudes herausgearbeitet (2.2), die zugleich Grundlage für die empirische Längsschnittanalyse darstellen. Bei der Ausarbeitung der Leitgedanken und der Analyse der Programme fokussiert dieser Artikel auf die wirtschaftliche Dimension des Neoliberalismus. Dieser Fokus dient ausschließlich einer detaillierteren Auseinandersetzung und stellt die weitergehende Wirkmächtigkeit des Neoliberalismus nicht in Frage. ${ }^{1}$

\subsection{Wirkungsgeschichte des Neoliberalismus}

Eine kontroverse Diskussion des Terminus Neoliberalismus findet nicht nur in Politik und Gesellschaft, sondern

1 Eine kompakte Übersicht hierzu findet sich u. a. in Butterwegge et al. (2008) und Harvey (2005). Ein Eindruck über die enorme Bandbreite neoliberaler Anschauungen entsteht bei einem Blick auf die kontrovers geführten Debatten, beispielsweise in den Bereichen Gesundheitswesen (Deppe 2005) oder Globalisierung (Altvater/Mahnkopf 2007). 
auch im akademischen Forschungsdiskurs statt. ${ }^{2}$ Hierbei reichen die Beiträge von teilweise fundamentaler Kritik (Saad-Filho/Johnston 2005; Butterwegge et al. 2008; Ötsch 2009; Crouch 2013; Mirowski 2013) bis hin zu einer Verteidigung bzw. Befürwortung marktliberaler Positionen (Fukuyama 1992; Willke 2003; Heuser 2003), obgleich sich die in der Minderheit befindenden BefürworterInnen aufgrund der mittlerweile überwiegend negativ vorherrschenden Begriffskonnotation nicht explizit als neoliberal ausweisen (Mirowski 2014).

Historisch betrachtet, reichen die Wurzeln des neuen Liberalismus bis in die 2oer-Jahre des vergangenen Jahrhunderts zurück, als es im Zuge der Weltwirtschaftskrise zu einer Diskreditierung wirtschaftsliberaler Werte kam. In dieser Zeit wandten sich politische Entscheidungsträger vermehrt staatsinterventionistischen Konzepten (USA, Großbritannien) zu, die von Keynes „General Theory“ (Keynes 1936/1964) inspiriert worden sind. Darüber hinaus erhielten auch politisch extreme Ideologien, wie der Kommunismus (UdSSR) und der Nationalsozialismus/Faschismus (Deutschland, Italien), starken Zulauf. Gleichwohl gab es eine kleine, aber international agierende Anhängerschaft (z. B. Ludwig Mises, Friedrich August Hayek, Walter Eucken, Alexander Rüstow, Louis Rougier, Walter Lippmann), die um die Erhaltung bzw. Neuausrichtung liberaler Ideen bemüht war. Auch wenn sich die einzelnen Vertreter auf gemeinsame inhaltliche Prinzipien verständigen konnten - bekannt ist v. a. das vom britischen Ökonomen Lionel Robbins ausgearbeitete Statement of Aims ${ }^{3}$

2 Infolge der historisch bedingten differierenden Verwendungsweise wird der Versuch einer exakten Begriffsbestimmung erschwert. So hat der Ausdruck im Laufe des 20. Jahrhunderts einen bemerkenswerten Bedeutungswandel erfahren. Ursprünglich 1938 von Alexander Rüstow auf dem Colloquium Walter Lippmann als Selbstbezeichnung für eine neue Wirtschaftsform in Abgrenzung zum Laissez-FaireLiberalismus des 19. Jahrhunderts vorgeschlagen, wurde er ab den 1980er-Jahren zunehmend im Zusammenhang mit kapitalismuskritischen Äußerungen in Verbindung gesetzt. Rund um die Jahrtausendwende erfuhr ihm insbesondere von seiner Gegnerschaft eine umfassende Kritik - so entwickelte er sich, von GegnerInnen als „negativer Inbegriff des entfesselten, global agierenden Kapitalismus" (Butterwegge et al. 2008: 14) hochstilisiert, schließlich zu einem politischen Kampfbegriff.

3 Dabei handelt es sich um ein sechs Punkte umfassendes Dokument, in dem neben Freiheit, Privateigentum an Produktionsmitteln, einer (internationalen) Marktordnung auch eine Redefinition der Staatsfunktion thematisiert werden (MPS online). als eine Art kurze programmatische Grundlage der MPS (Walpen 2000) -, so sind diese jedoch nicht als homogener Teilnehmerkreis zu verstehen. Während etwa die Vertreter des Ordoliberalismus die Notwendigkeit einer politischen Organisation des Marktwettbewerbs in Form einer Wirtschaftsverfassung (Eucken 1952/1990) sahen, betont Hayek in seinen späteren Werken die Rolle evolutionär gewachsener Strukturen (Hayek 1969, 1979/1996).

Aufgrund der allgemeinen Abkehr von marktliberalen Anschauungen wird im Zuge der Auseinandersetzung mit der neoliberalen Hegemonieentfaltung der Zeitraum bis etwa 1947 als „Verteidigungs- und Konsolidierungsphase“ (Plehwe/Walpen 1999: 20) charakterisiert, in der es vorrangig (z. B. auf dem Lippmann-Colloquium 1938) um den Zusammenschluss „zahlenmäßig unterlegener Truppen“ (Nash 1976: 26) ging. Mit der nach dem Zweiten Weltkrieg einsetzenden „Golden Age-Phase“ (van Treeck et al. 2007: 637) des Kapitalismus, die u. a. durch ein starkes wirtschaftspolitisches Engagement des Staates geprägt war, wurde auf dem Gründungstreffen der MPS parallel dazu die Bewegungsphase des Neoliberalismus eingeläutet (Plehwe/Walpen 1999). Ausgehend von der Zielsetzung einer Veränderung des gesellschaftlichen Meinungsklimas zugunsten des Neoliberalismus, entwickelte sich Hayeks Elitentheorie (Hayek 1949/1960) zur gezielten Beeinflussung der gesellschaftlichen Meinung durch eine kleine Gruppe intellektueller Vordenker (original thinkers), deren Konzepte mittels Ideenverbreiter (secondhand dealers in ideas) unter die Bevölkerung zu transferieren sind, zur langfristigen strategischen Ausrichtung der MPS. Als praktische Konsequenz dieser Überlegungen wurde in den nächsten Jahrzehnten ein internationales Netzwerk an neoliberalen Thinktanks errichtet. Diesen wurde die Aufgabe der institutionell organisierten Ideenverbreitung zugewiesen und sie übernahmen in weiterer Folge einen entscheidenden Anteil bei der Etablierung marktliberaler Anschauungen (Plehwe/ Walpen 1998; Ötsch et al. 2018). ${ }^{4}$

Sieht man von dem kurzen Abschnitt des als Soziale Marktwirtschaft bezeichneten ordoliberalen

$4 \quad$ Mit dem Institute of Economic Affairs, welches an der Umorientierung der englischen Tory Party beteiligt war (Cockett 1995) und der Heritage Foundation, deren Mandatefor-Leadership-Dokument Reagans Angebotspolitik vorwegnahm (Gellner 1995), gelang es, marktliberales Gedankengut auf die wirtschaftspolitische Ebene zu übertragen. 
Intermezzos in der BRD zu Beginn der 1950er-Jahre ab (Ptak 2004), wird der neoliberalen Ideologie auf theoretischer, aber v. a. auf wirtschaftspolitischer Ebene bis Ende der 1960er-Jahre ein geringer Einfluss bescheinigt. Erst eine Kette an Ereignissen im Laufe der 1970er-Jahre begünstigte die allmähliche Herbeiführung des neoliberalen Transformationsprozesses in westlichen Industrienationen. Kowall (2013) führt in diesem Zusammenhang fünf Erklärungsansätze für die Ablösung der keynesianisch geprägten Wirtschaftsordnung an: (i) Die politikökonomische Erklärung thematisiert die falsche Reaktion des Staates auf die durch den Ölpreisanstieg entstandene Lohn-PreisSpirale, wodurch es zum Auftreten der Stagflation kam; (ii) gemäß der klassenspezifischen Erklärung wurde zu dieser Zeit die Nachkriegsallianz zwischen Realkapitalisten und Gewerkschaftsorganisationen zugunsten eines neuen Bündnisses zwischen Finanzund Realkapitalisten aufgekündigt; (iii) die ökologische Erklärung hebt die natürlichen Grenzen des keynesianischen Wachstumsmodells hervor, welche durch die Ölkrise offenkundig wurden; (iv) die Auflösung des Bretton-Woods-Systems 1973 wird in der finanzwissenschaftlichen Erklärung als der Beginn einer neuen - von Finanzmärkten dominierten Wirtschaftsordnung interpretiert; (v) und schließlich unterstreicht die ideengeschichtliche Erklärung, dass die Veränderung des Meinungsklimas seitens der neoliberalen Theoretiker gezielt vorbereitet wurde. ${ }^{5}$ Die neoliberale Wende entstand also im Zusammenspiel einer großen realwirtschaftlichen Veränderung (i-iv) und einer bewusst intendierten Strategie zur Hegemoniegewinnung (v).

Die endgültige Ablösung der keynesianischen Wirtschaftsepoche wurde in den späten 1970er-Jahren durch eine wirtschaftspolitische Umwälzung (Chile, Großbritannien, USA, BRD) vollzogen. Etwa zur selben Zeit setzte auch ein Bedeutungswandel der ursprünglich positiv intendierten Begriffskonnotation ein, da KritikerInnen in Chile die Reformen unter Augusto Pinochet unter der Bezeichnung neolibera-

5 Auch die Etablierung des von der schwedischen Reichsbank gestifteten Alfred-Nobel-Gedächtnispreises für Wirtschaftswissenschaften verhalf dem Neoliberalismus salonfähig zu werden, da die beiden ersten Vorsitzenden des Entscheidungskomitees Erik Lundberg (1975-79) und Assar Lindbeck (1980-94) Mitglieder der MPS waren. In deren Amtsperioden erhielten gleich sieben Personen aus dem MPS-Netzwerk die Auszeichnung. lismo zusammenfassten. ${ }^{6}$ Sie verbanden damit eine minimalstaatliche, marktfundamentale bzw. -radikale Variante des Kapitalismus auf Kosten des sozialen Sicherheitsgefüges. Diese Deutung setzte sich im Zuge der Amtsübernahme von Thatcher und Reagan im Wesentlichen auch im englischsprachigen Raum durch (Boas/Gans-Morse 2009).

Trotz dieser Kritik erhielten neoliberale Positionen zu Beginn der 1990er-Jahre weiteren Aufschwung. Mitverantwortlich hierfür war das als Washington Consensus bezeichnete wirtschaftspolitische Maßnahmenbündel, welches von Weltbank und Internationalem Währungsfonds als Leitlinie für die Vergabe von Krediten an Entwicklungsländer - v. a. in Lateinamerika herangezogen wurde (Williamson 1993). Zudem erhielt der Neoliberalismus nach dem Ende des Systemkonflikts durch die Selbstauflösung der UdSSR im Dezember 1991 den Status einer ideologischen Führerschaft (Butterwegge et al. 2008). Aktuell haben neoliberale Anschauungen in sozial- und wirtschaftspolitischen Diskursen eine gewichtige Position, da sich mitunter auch gegenüber dieser Strömung zunächst reservierte politische Kräfte marktliberalen Standpunkten angenähert haben (Kapeller/Huber 2009; Mirowski/Plehwe 2009; Nachtwey 2009; Ötsch et al. 2018).

Auch in Österreich war ab Mitte der 1980er-Jahre ein Wandel in Richtung einer stärker angebotsorientierten Wirtschaftspolitik zu beobachten. Die wirtschaftspolitischen Maßnahmen infolge der durch den Erdölpreisschock ausgelösten Rezession folgten jedoch zunächst einer anderen Maxime. Während viele westliche Industriestaaten ihr Hauptaugenmerk auf die Inflationsbekämpfung und ein ausgeglichenes Budget legten, wurde in Österreich mit dem Austrokeynesianismus die Strategie einer aktiven staatlichen Wirtschaftsgestaltung verfolgt, wobei dem (Voll-) Beschäftigungsziel Priorität zugeschrieben wurde (Lacina 1993). ${ }^{7}$ Diese Phase wurde maßgeblich durch

6 Im Anschluss an die politische Revolution im September 1973 nahm das Regime unter Pinochet mit maßgeblicher Unterstützung von Friedmans Chicago Boys die wirtschaftliche Umstrukturierung Chiles vor. So wurden u. a. fast alle Preiskontrollen aufgehoben, zahlreiche Staatsbetriebe sowie weite Teile des Gesundheits- und Rentensystems privatisiert, die Staatsausgaben um etwa ein Viertel reduziert sowie eine massive Zinserhöhung betrieben (Hira 1998).

7 Der Terminus Austrokeynesianismus wurde im Nachhinein vom österreichischen Ökonomen Hans Seidel als Bezeichnung für die wirtschaftspolitischen Maßnahmen Österreichs von Mitte der 7oer- bis Mitte der 8oer-Jahre geprägt (Kriechbaumer 2006). 
die Sozialdemokratie unter der Kanzlerschaft Kreiskys (1970-1983) geprägt. Im Wesentlichen beruhte das Modell auf drei Säulen: (i) expansive Budgetpolitik (deficit spending); (ii) Hartwährungspolitik; sowie (iii) sozialpartnerschaftliche Partizipation (Rieger 1993). Das System des Austrokeynesianismus geriet zu Beginn der 1980er-Jahre allmählich in die Defensive, was u. a. auf die teilweise Unrentabilität der verstaatlichten Betriebe, eine wachsende Staatsschuldenquote und die Veränderung des innerpolitischen Kräfteverhältnisses zurückzuführen ist.

\subsection{Leitgedanken des neoliberalen Gedanken- gebäudes}

Auf theoretischer Ebene erweist sich das Bestreben, eine genaue Charakterisierung des Neoliberalismus vorzunehmen, nicht nur wegen dessen begrifflicher Bedeutungsveränderung, sondern auch aufgrund seiner inhaltlichen Bandbreite, als schwieriges Unterfangen. Das neoliberale Gedankengebäude ist als Konglomerat (teilweise) heterogener Denkschulen mit länderspezifischen Ausprägungen (z. B. Österreichische Schule, Freiburger Schule, Chicago School) anzusehen und besticht durch eine große Wandlungsfähigkeit. Nichtsdestoweniger fungierten in seiner Entwicklungsgeschichte gewisse wiederkehrende Leitgedanken als theoretisches Bindeglied zwischen den verschiedenen Ausprägungen. Da diese in weiterer Folge auch als Orientierungspunkt für die empirische Analyse verwendet werden, fokussiert sich die Aufzählung vermehrt auf wirtschaftspolitische Aspekte:

- (L1) Ablehnung kollektivistischer Strömungen, wobei hierunter sowohl Nationalsozialismus und Kommunismus/Sozialismus als auch Keynesianismus und Wohlfahrtsstaat subsumiert werden (Butterwegge et al. 2008).

- (L2) Wettbewerbsmarkt als wichtigste Koordinationsinstanz für ökonomische Aktivitäten wird zur Legitimierung einer marktdominierten Gesellschaft herangezogen (Nordmann 2005).

- (L3) Redefinition der Funktion des Staates im Sinne der Durchsetzung und Sicherung der marktwirtschaftlichen Funktionsfähigkeit ${ }^{8}$ (Walpen 2000).

8 Das neoliberale Staatsverständnis ist ein ambivalentes. Einerseits wird bei sozialstaatlichem Engagement ein schwacher Staat beabsichtigt, andererseits bedarf die Organisation der Marktgesellschaft eines starken Staats. Die Forderung nach staatlichen Rahmenbedingungen wird von Eucken
- (L4) Orientierung an einer global ausgerichteten Standortpolitik, die ökonomisches Wachstum durch eine Verbesserung marktwirtschaftlicher Anreize sowie durch Abbau von Handelsbarrieren (Freihandel) und internationalen Standards (Deregulierung) zu generieren versucht (Clement et al. 2012; Butterwegge et al. 2008).

- (L5) Wirtschaftliche Handlungen werden ausnahmslos auf individuelle Verhaltensweisen (Einstellungen, Ziele etc.) zurückgeführt (Hayek 1980). ${ }^{9}$ In diesem Kontext wird die Selbstverantwortlichkeit des Individuums hervorgehoben.

- (L6) Ablehnung wohlfahrtsstaatlicher Verteilungsmaßnahmen, die über die Existenzsicherung hinausgehen. ${ }^{10}$ Ein ausgedehnter Sozialstaat wird im internationalen Wettbewerb als Standortnachteil wahrgenommen (Klages/Strutynski 1997).

\section{Methodische Konzeption}

Um empirisch zu überprüfen, in welchem Ausmaß neoliberale Positionen in die parteiprogrammatische Ausgestaltung österreichischer Parteien eingeflossen sind, wird im Folgenden eine vergleichende Längsschnittanalyse durchgeführt. Als Datengrundlage dienen Grundsatzprogramme von ÖVP, SPÖ, FPÖ und Grünen. Die Auswahlbasis bildet ein breites politisches Spektrum ab, innerhalb dessen christlich-konservative, sozialdemokratische, liberale und ökologische Werte anzutreffen sind. Ein Indiz für eine neoliberale Deutungshoheit liegt vor, wenn die Grundwerte und Zielsetzungen des Neoliberalismus über alle Parteien und Ideologien hinweg Verbreitung finden. Eine auf diese Weise resultierende Angleichung der programmati-

pointiert formuliert: „Staatliche Planung der Formen - ja; staatliche Planung und Lenkung des Wirtschaftsprozesses nein“ (Eucken 1951: 72). Folglich wird nicht die Zerschlagung staatlicher Macht, sondern vielmehr eine Umwandlung vom Wohlfahrts- zum Wettbewerbsstaat angestrebt.

9 Kollektive Systeme (Gesellschaft) werden nicht als eigenständige Akteure anerkannt. So ist für Hayek die Gesellschaft „keine handelnde Person, sondern eine geordnete Struktur von Handlungen, die sich daraus ergibt, dass ihre Mitglieder gewisse abstrakte Regeln beachten" (Hayek 1980: 131).

10 Hayek sieht in der zunehmenden Verteilungspolitik des Wohlfahrtsstaates eine Aushöhlung der kapitalistischen Wirtschaftsordnung. „Das Soziale bezeichnet kein definierbares Ideal, sondern dient heute nur mehr dazu die Regeln der freien Gesellschaft, der wir unseren Wohlstand verdanken, ihres Inhalts zu berauben“ (Hayek 1979/1996: 16). 
Tabelle 1: Grundsatzprogramme für die empirische Analyse ${ }^{11}$

\begin{tabular}{lll}
\hline Fraktion & \multicolumn{1}{c}{$\begin{array}{c}\text { Programmatik aus der } \\
\text { neoliberalen Epoche }\end{array}$} & \multicolumn{1}{c}{$\begin{array}{c}\text { Vergleichsprogrammatik } \\
\text { (aus der Golden-Age-Phase) }\end{array}$} \\
\hline ÖVP & 2015 & 1972 \\
SPÖ & 1998 & 1978 \\
FPÖ & $2005^{12}$ & 1973 \\
Grüne & 2001 & $1986^{13}$ \\
\hline
\end{tabular}

Quelle: (Eigene Darstellung)

schen Inhalte käme auch Hayeks Vorstellung von einer dominierenden Weltanschauung (Elitentheorie) sehr nahe, hierbei ist auf die - eingangs erwähnte - Primärfunktion von Grundsatzprogrammen als abstrakte Leitlinienvorgabe und die damit verbundene eingeschränkte Relevanz für tagespolitische Entscheidungen zu verweisen.

Im Sinne des vergleichenden Designs wurde das jeweils aktuelle Parteiprogramm einem früheren Programm, welches noch nicht in die vom Neoliberalismus gekennzeichnete Epoche fällt, gegenübergestellt (Tabelle 1).

Der vergleichenden empirischen Textanalyse wird die Methodik der qualitativen Inhaltsanalyse nach Mayring (2010) zugrunde gelegt. Die Strategie zur Entwicklung der Untersuchungsdimensionen orientierte sich dabei einerseits am Datenmaterial (Parteiprogrammatiken), andererseits wurden auch die sechs Leitgedanken des Neoliberalismus (siehe: 2.2) in die provisorische Kategorienfestlegung miteinbezogen.

11 Aufgrund einer notwendigen Beschränkung auf die hier interessierenden Zeitphasen wurde auf die Analyse der frühen Parteiprogramme (von vor ca. 1970) verzichtet. Dies galt parteibezogen für die Programme aus den Jahren: ÖVP $(1945,1952,1958,1965)$, SPÖ $(1947,1958,1966)$, FPÖ $(1955,1957 / 58,1964,1968 / 70)$.

12 Da das aktuelle Programm aus 2011 eine stark gekürzte, aber inhaltlich nur minimal modifizierte Version von 2005 ist, wurde auf diese umfangreichere Variante zurückgegriffen. Ebenso verzichtet wurde auf die Analyse der Programmatik von 1985 als frühe und damit später (2005) überholte Variante der neoliberalen Epoche. Selbiges gilt für die frühe Programmatik der Grünen (1990).

13 Wenngleich aufgrund der jungen Parteigeschichte auf keine früheren Schriften zurückgegriffen werden konnte, scheint eine Verfälschung der Ergebnisse durch eine neoliberale Einflussnahme nicht gegeben zu sein, da sich die Partei als (ökologische) Alternative zur bestehenden Gesellschaftsund Wirtschaftsordnung versteht.
Insofern ist die hier gewählte Herangehensweise eine Mischform zwischen induktivem und deduktivem Ansatz. Im Anschluss an die provisorische Einteilung wurde eine am Datenmaterial ausgerichtete Kategorienrevision durchgeführt und folgende finale dimensionale Einteilung festgelegt: ${ }^{14}$

(i) In der Dimension Marktbild soll die grundsätzliche Einstellung zur marktbasierten Organisationsform erhoben werden. Dabei interessiert v. a., inwiefern die Zuschreibung bestimmter Wesensmerkmale mit der oben angeführten marktoptimistischen Sichtweise des Neoliberalismus, im Sinne der zentralen Lenkungsfunktion ökonomischer Aktivitäten (L2), Übereinstimmungen aufweist.

(ii) In der Dimension Wirtschaftspolitik werden darauf aufbauend die verschiedenen Zielsetzungen und Maßnahmen wirtschaftspolitischen Handelns erfasst. Maßgebliche Kennzeichen für eine neoliberale Handschrift sind dabei das Bestreben der langfristigen Gewährleistung einer global ausgerichteten Marktgesellschaft $\left(\mathrm{L}_{3}\right)$ infolge einer stetigen Optimierung von (angebotsseitigen) Anreizmechanismen (L4).

(iii) In der Dimension Menschenbild werden die Grundwerte und Bedingungen der menschlichen Selbstverwirklichung im Spannungsfeld zwischen individualistischen und kommunitaristischen Idealen thematisiert. Der neoliberalen Position liegt hierbei ein stark individualistisch geprägtes Menschenbild vom selbstverantwortlichen Individuum (L5) zugrunde.

14 Im Zuge der Revision wurde etwa $50 \%$ des Datenmaterials anhand der provisorischen Einteilung gesichtet. Dabei konnten zum neoliberalen Leitgedanken der Ablehnung des Kollektivismus kaum verwertbare Textpassagen identifiziert bzw. die vorhandenen Elemente nicht sinnvoll von anderen Dimensionen getrennt werden. Die Eliminierung dieser Dimension war somit naheliegend. 
(iv) In der Dimension Wohlfahrtsstaat erfolgt eine Sondierung sowohl im Hinblick auf die Einstellung zu Höhe und Reichweite wohlfahrtsstaatlicher Leistungen als auch in Bezug auf die Beurteilung verteilungspolitischer Maßnahmen. Gemäß der neoliberalen Position schmälert ein umfassender Sozialstaat den individuellen Leistungsanreiz und führt im internationalen Vergleich zu Standortnachteilen (L6).

\section{Ergebnisdarstellung der empirischen Untersuchung}

Die Resultate der empirischen Abhandlung werden in vier Subkapiteln vorgestellt, wobei in jedem Teilstück eine Partei in der dimensionalen Reihenfolge Marktbild - Wirtschaftspolitik - Menschenbild - Wohlfahrtsstaat behandelt wird.

\subsection{Interpretation der Parteiprogramme der ÖVP}

Nach der Niederlage bei der Nationalratswahl (NRW) 1970 ging die ÖVP erstmals in die Opposition und verblieb dort auch nach den vorgezogenen Wahlen 1971. Infolgedessen kam es unter Obmann Schleinzer zu einer Grundsatzdebatte, deren Ergebnisse im Salzburger Programm (Beschluss: 01.12.1972) festgeschrieben wurden (ÖVP 2018 online). Darin verortet sie sich als „Partei der fortschrittlichen Mitte“ (ÖVP 1972: 4). Die am 37. Bundesparteitag (12./13.05.2015) in Wien beschlossene aktuelle Programmatik war das Resultat des im März 2014 gestarteten Projekts Evolution Volkspartei. Das Selbstverständnis orientiert sich v. a. an den Prinzipien der Freiheit und Eigenverantwortung (ÖVP 2015: 5). Realpolitisch befand man sich zu jener Zeit in einer Koalition mit der SPÖ und stellte mit Mitterlehner den Vizekanzler.

\subsubsection{Marktbild}

Die ÖVP vermittelt in ihrer Programmatik von 1972 ein optimistisches Bild von der als soziale Marktwirtschaft bezeichneten Wirtschaftsform, die mit einer prosperierenden Gesellschaftsentwicklung einhergeht (ÖVP 1972: 13f). Demnach führen die in der Marktwirtschaft innewohnenden Kräfte, in Verbindung mit einer produktivitätsorientierten Wirtschaftspolitik, zu gesamtwirtschaftlichem Wohlstand und sozialer Sicherung:

„Instrument dieses Strebens ist die soziale Marktwirtschaft, die in ihrer Weiterentwicklung nicht allein wachsenden Wohlstand erringen und individuelle Bedürfnisse befriedigen, sondern auch die gemeinschaftlichen Aufgaben in Gegenwart und Zukunft erledigen soll" (ÖVP 1972: 21).

Das kapitalistische System wird allerdings nicht unter rein ökonomischen Gesichtspunkten wahrgenommen, sondern auch für den Aufbau wohlfahrtsstaatlicher Infrastruktur dienstbar gemacht. Um die Vorzüge der sozialen Marktwirtschaft realisieren $\mathrm{zu}$ können, wird die Notwendigkeit staatlicher Rahmenbedingungen (z. B. freie Berufswahl, freier Wettbewerb) sowie die Mitwirkung der österreichischen Sozialpartnerschaft vorausgesetzt (ÖVP 1972: 21f).

Das Programm von 2015 schließt an diese grundsätzlich marktoptimistische Sichtweise an, wenngleich Veränderungen in der Akzentuierung festgestellt werden können. So wird die etwas defensiv klingende Formulierung in Bezug auf die Fähigkeit des Wirtschaftsmodells in der 72er-Programmatik zugunsten einer offensiven Ausrichtung eines selbstregulierenden Systems ersetzt. Ferner hielten auch ökologische Themenfelder stärker Einzug in die aktuelle Programmatik:

„Die Ökosoziale Marktwirtschaft setzt den richtigen

Rahmen und die richtigen Anreize, um Innovationskraft, Wachstum, Wohlstand und soziale Sicherheit mit einer nachhaltigen klima- und umweltschonenden Entwicklung $z u$ vereinbaren. Wir vertreten die Ökosoziale Marktwirtschaft offensiv als wegweisendes politisches Leitbild für Europa und die Welt" (ÖVP 2015: 14).

Im neuen Grundsatzprogramm wird zudem die Bedeutung der, auf einem Höchstmaß an wirtschaftlicher Freiheit beruhenden, unternehmerischen Leistungsfähigkeit als „Fundament unseres Gesellschaftsmodells“ (ÖVP 2015: 6) herausgestrichen. Soziale und ökologische Herausforderungen werden stets vor dem Hintergrund der internationalen Wettbewerbsfähigkeit betrachtet. Mit dieser Vorstellung ist auch eine ausgeprägte Ablehnung gegenüber staatlichen Regulierungsmaßnahmen verbunden (ÖVP 2015: 10, 18).

\subsubsection{Wirtschaftspolitik}

Die konkrete Ausgestaltung der wirtschaftspolitischen Maßnahmen im 72er-Programm ist dem Leitbild der sozialen Marktwirtschaft verpflichtet. Hierbei wird die Bedeutung der unternehmerischen Leistungsfähigkeit hervorgehoben, um sich im internationalen Konkurrenzkampf zu profilieren (ÖVP 1972: 21). Die Zielvorgaben besitzen zudem eine gesellschaftspolitische Komponente, bei der auch das Interesse der Arbeit- 
nehmerInnen in Form der Beschäftigungssicherung Berücksichtigung findet:

„Diese Entscheidungen [der Wirtschaftspolitik Anm. des Autors] müssen sich den Zielen der sozialen Marktwirtschaft, wie Stabilität des Geldwertes, wirtschaftliches Wachstum, Sicherung der Vollbeschäftigung und Erhaltung des notwendigen Leistungsanreizes und Sparwillens unterordnen" (ÖVP 1972: 22f).

Das staatliche Instrumentarium zur Wahrnehmung dieser Aufgaben beruht auf einem auf Basis einer wirtschaftsnahen Gesetzgebung entsprechenden Policy-Mix (z. B. Steuer-, Konjunktur- und Investitionspolitik) (ÖVP 1972: 22). Staatlicher Dirigismus wird hingegen mit dem Verweis steigender Abhängigkeiten von Unternehmen und BürgerInnen abgelehnt (ebd.: 11, 15).

In der gegenwärtigen Schrift ist das wirtschaftspolitische Handeln des Staates an internationaler Wettbewerbsfähigkeit ausgerichtet. Insofern fokussieren sich die Maßnahmen in der Hauptsache auf eine Stärkung der Angebotsseite. Angestrebt werden u. a. ein leistungsfreundliches Steuersystem als Anreizmechanismus für Privatinitiative und Investitionsbereitschaft sowie mehr (Arbeitsmarkt-)Flexibilität (ÖVP 2015: 21, 24). Ferner erkennbar ist ein Staatsskeptizismus, der sich sowohl auf (Regulierungs-)Vorschriften als auch auf die staatliche Leistungserbringung bezieht:

„Eine automatische Auslaufklausel für Gesetze und Verordnungen [Sunset-Legislation] soll beschlossene Maßnahmen kontinuierlich auf ihre Wirksamkeit und Sinnhaftigkeit überprüfen. Wo - insbesondere wirtschaftliche - Leistungen besser durch Private erbracht werden, setzen wir uns für die Privatisierung staatlicher Unternehmungen ein, die nicht der Daseinsvorsorge dienen. Die Gewährleistung von Leistungen durch den Staat bedeutet nicht, dass er diese selbst erbringen muss" (ÖVP 2015: 18). ${ }^{15}$

Die Partei vertritt die Sichtweise eines schlanken Staatswesens, dessen - an unternehmerischen Prinzipien ausgerichteten - Handlungen eine Maximierung individueller Wahlmöglichkeiten und eine sparsame Ressourcenverwendung ermöglichen soll (ÖVP 2015: 14).

\subsubsection{Menschenbild}

Das Menschenbild in der Programmatik von 1972 baut auf zwei leitenden Gedanken auf: Solidarismus und

15 Private Anbieter können durchaus Aufgaben in der Daseinsvorsorge (z. B. Verkehrswesen, Gas-, Wasser- und Elektrizitätsversorgung) übernehmen und Profite generieren. Der Staat übernimmt lediglich die Versorgungsgarantie.
Subsidiarität. Dem solidarischen Prinzip zufolge ist die individuelle Selbstverwirklichung nur im Wechselspiel mit der Gemeinschaft realisierbar:

„Der Mensch kann nur in der Gemeinschaft zur vollen Entfaltung seiner Persönlichkeit kommen. Er hat die Verpflichtung, seinen Beitrag zum Gemeinwohl zu leisten. Die Gesellschaft ihrerseits hat die Verpflichtung, die Selbstverwirklichung des Menschen zu ermöglichen"(ÖVP 1972: 7).

Daneben werden mit dem subsidiären Prinzip individuelle Freiheit und Selbstbestimmung als maßgebliche Faktoren für eine erfolgreiche Entwicklung hervorgehoben. Staatliche Maßnahmen müssen so gestaltet sein, dass sie nicht im Sinne eines uniformierenden Charakters gegen die persönliche (Handlungs-)Freiheit gerichtet sind, sondern leistungsfördernde Anreize aufweisen (ÖVP 1972: 7, 9).

Bei der Ausgestaltung der menschlichen Arbeitswelt spielt neben dem Leistungsgedanken die humane Selbstverwirklichung eine wichtige Rolle. Auch wird vor einem übersteigerten Leistungszwang und einer Entfremdung des Menschen infolge technischer Spezialisierung gewarnt (ebd.: 9f., 18f).

Im aktuellen Grundsatzprogramm wurde das ausgewogene Verhältnis zwischen Solidarität und Subsidiarität aus dem 72er-Dokument zugunsten einer Stärkung der individuellen Freiheit aufgegeben. Die solidarische Komponente beschränkt sich nun auf den Hinweis der Verantwortung gegenüber der Gemeinschaft, wobei hierunter v. a. die Familie verstanden wird (ÖVP 2015: 9f.). An erster Stelle wird das eigenverantwortliche Lösen aller Angelegenheiten befürwortet. Diese Maxime bildet gemeinsam mit der Forderung nach der Abwesenheit staatlicher Anordnungen die zentrale Bedingung zur freien Persönlichkeitsentfaltung (ebd.: 5, 10):

„Wir stehen für eine Gesellschaftsordnung, die dem

Einzelnen bestmögliche Entfaltung durch ein Höchstmaß an

Wahlfreiheit ermöglicht. Wie Menschen ihr Leben gestalten

(...) muss in ihrer freien Entscheidung liegen" (ÖVP 2015: 25).

Ein weiterer Unterschied zur Vorgängerprogrammatik lässt sich in Bezug auf die Ausgestaltung der menschlichen Arbeitswelt erkennen, wo der Leistungsgedanke zum dominierenden Prinzip aufgestiegen ist, während Formulierungen zu humanen Perspektiven vage gehalten sind (ÖVP 2015: 11, 21f.).

\subsubsection{Wohlfahrtsstaatliches Engagement}

Die ÖVP weist in ihrer früheren Programmatik auf den dynamischen Charakter wohlfahrtsstaatlicher 
Maßnahmen hin, die sich an den jeweiligen sozioökonomischen Gegebenheiten orientieren müssen. Dabei bekennt sie sich zum sozialen Staat in einer partnerschaftlichen Gesellschaft, dessen Leistungen mehr als die reine materielle Existenzsicherung abzudecken haben:

„Eine moderne Sozialpolitik muss außer der quantitativen Vorsorge auch einen qualitativen Beitrag zur Verbesserung der Lebensverhältnisse des einzelnen leisten" (ÖVP 1972: 24).

Zugleich warnt die Partei vor den Folgewirkungen, die von einem etablierten Sozialsystem in Form von Gewöhnungseffekten sowie stetig steigenden Finanzaufwendungen ausgehen können. Staatliche Sozialpolitik ist daher durch zumutbare eigenständige private Vorsorgeleistungen zu ergänzen (ÖVP 1972: 25).

Die Verteilung der personellen Einkommen soll grundsätzlich durch das Leistungsprinzip geregelt sein, um Leistungsbereitschaft, Fortschritt, aber auch sozialstaatliche Sicherheit zu ermöglichen. Aus der nicht vollkommen praktizierten Leistungsvergütung ergeben sich finanzielle Mittel, die als sozialstaatliche Unterstützung für Nichtleistungsbefähigte herangezogen werden (ebd.: gf.).

Im Programm von 2015 begreift die Partei wohlfahrtsstaatliches Engagement primär als eine zeitlich zu begrenzende staatliche Unterstützungsleistung, die im Falle von Notlagen oder in bestimmten Lebensabschnitten (z. B. Pension) zum Tragen kommt. Die Definition von Lebensrisiken obliegt dabei dem Sozialstaat. Leistungen müssen so gestaltet werden, dass sie keine andauernden Abhängigkeitsverhältnisse erzeugen, sondern eigenverantwortliches Handeln fördern:

„Die sozialstaatliche Grundversorgung in Schicksalsfällen ist ebenso unverzichtbar wie der Beistand in bestimmten Lebensphasen. Unterstützungen Einzelner oder sozialer Institutionen sind im Sinn der Stärkung von Eigenverantwortung und Selbstermächtigung auf Zeit anzulegen" (ÖVP 2015: 31).

Gemäß der Eigenverantwortung wird von den Individuen daher die Pflicht zur privaten Vorsorge eingefordert. Der "solidarische“ Wohlfahrtsstaat ist in einem tauschtheoretischen Sinne zu verstehen, bei dem nicht nur Solidarität mit den LeistungsempfängerInnen, sondern auch mit den LeistungsfinanziererInnen hervorgehoben wird (ÖVP 2015: 12).

Verteilungspolitische Maßnahmen des Staates werden mit dem Verweis negativer Effekte auf den gesamtwirtschaftlichen Wohlstand abgelehnt (ebd.: 30).

\subsection{Interpretation der Parteiprogramme der SPÖ}

Die Programmatik von 1978 (Beschluss: 20.05.1978) entstand während der Hochphase der SPÖ, die von 1971 bis 1983 unter der Kanzlerschaft von Kreisky alleinregierende Partei war. Inhaltlich ist das Programm an den Gedanken der sozialen Demokratie und einer Gesellschaft ohne Klassengegensätzen ausgerichtet, wie es in der Vorbemerkung des Dokuments heißt (SPÖ 1978: 3). Im Vergleich dazu wurde das aktuelle Programm am Ende einer langen Koalitionsphase mit der ÖVP als Juniorpartner (1986-1999), die 1999 unter dem Parteivorsitz von Klima endete, beim Parteitag am 30./31. Oktober 1998 beschlossen. Dementsprechend war diese Schrift Ausdruck einer inhaltlichen Modernisierung und Öffnung der Partei in Richtung Marktkonformität.

\subsubsection{Marktbild}

Im Parteiprogramm von 1978 herrscht eine negative Auffassung vom kapitalistischen Wirtschaftssystem vor, das durch zwei wesentliche Merkmale gekennzeichnet ist. Erstens ist das System von Gewinnstreben und Konkurrenz geprägt. Dabei stehen die Interessen der Eigentümer und Unternehmer und nicht die der Gesamtbevölkerung im Vordergrund. So üben die Produktionsbedingungen schädigende Einflüsse auf Mensch und Gesellschaft aus, da nicht deren Bedürfnisbefriedigung, sondern ökonomische Faktoren wie unternehmerischer Profit und Wachstum die treibenden Kräfte des Wirtschaftssystems darstellen. Menschen, die in diesem Konkurrenzsystem nicht bestehen können, werden an den Rand gedrängt (SPÖ 1978: 11).

Der zweite Kritikpunkt wendet sich gegen die Annahme der Stabilität der kapitalistischen Marktwirtschaft. Vielmehr wird dem System eine immanente Tendenz zur Krisenanfälligkeit attestiert, demzufolge wirtschaftliche Instabilitäten kein Resultat eines Staatsversagens sind, sondern aufgrund von Marktversagen auftreten:

„Deshalb stellt sich den Sozialisten nach wie vor die historische Aufgabe, der konservativen Illusion von den Selbstheilungskräften der Marktwirtschaft eine klare Analyse der wirklichen Krisenursachen entgegenzustellen" (SPÖ 1978: 12).

Der Kapitalismus muss durch aktives politisches Handeln gestützt werden, wenngleich dadurch die Kri- 
senanfälligkeit des Systems nicht überwunden werden kann (SPÖ 1978: 10f.).

Im Vergleich zur Sichtweise von 1978 offenbart das neue Parteiprogramm ein weitaus optimistischeres Marktbild. Während zuvor noch die systemische Krisenanfälligkeit als wesentliches Merkmal herausgestellt wurde, wird nun stärker auf Marktkräfte und ihre koordinierende Fähigkeit zur effizienten Leistungserstellung vertraut. In diesem Zusammenhang wird eine funktionierende Volkswirtschaft als Voraussetzung für Vollbeschäftigung, wirtschaftlichen Wohlstand und soziale Sicherheit angesehen:

„Märkte - ihre Dynamik und Innovationsfähigkeit - liefern innerhalb definierter Rahmenbedingungen und bei fairem Wettbewerb einen wichtigen Beitrag zur Förderung des Wohlstands durch ihren Zwang zu effizienter und preiswerter Erbringung von Leistungen im Interesse der Verbraucherinnen und Verbraucher" (SPÖ 1998: 6f.).

Die Sozialdemokraten sehen die Grenzen des Marktmechanismus jedoch insbesondere bei verteilungspolitischen Fragestellungen erreicht, welche ein politisches Eingreifen verlangen (SPÖ 1998: 7).

\subsubsection{Wirtschaftspolitik}

Angesichts der Fehlentwicklungen des Kapitalismus wird eine umfassende Transformation des Wirtschaftssystems nach sozialistischen Prinzipien gefordert, wobei bei der Umsetzung dem gemeinwirtschaftlichen Sektor in Gestalt verstaatlichter Unternehmen und öffentlicher Versorgungsbetriebe eine zentrale Rolle zukommt. Die betriebliche Leistungserstellung soll im Rahmen eines planenden Wirtschaftsprozesses und nach demokratischen Grundprinzipien im Interesse der gesamtgesellschaftlichen Wohlstandsvermehrung organisiert werden (SPÖ: 1978: 23, 29):

„Welchen Beitrag ein großer gemeinwirtschaftlicher

Sektor, vor allem die Verstaatlichung der Grundindust-

rie und Großbanken, zur Erhaltung der wirtschaftlichen

Unabhängigkeit und zur Sicherung der Vollbeschäftigung

leisten kann, hat sich gerade in Österreich gezeigt" (SPÖ 1978: 23).

Neben dem Bekenntnis zu einem starken öffentlichen Wirtschaftssektor werden dem Staat weitreichende Gestaltungskompetenzen eingeräumt, um die negativen Folgen des Kapitalismus einzudämmen. Expliziten Vorrang hat dabei die Schaffung und Sicherung einer humanen Arbeitswelt (Vollbeschäftigung). Um ein möglichst hohes Niveau zu erhalten, werden öffentliche Investitionen zur Nachfragestützung sowie
Steuersenkungen zugunsten der Kaufkraftstärkung unterer und mittlerer Einkommensschichten forciert (SPÖ 1978: 24f.).

Zwanzig Jahre später wird mit der wirtschaftspolitischen Ausrichtung in der 78er-Programmatik teilweise gebrochen. Zwar wird dem Staat bei der Erbringung von öffentlichen Leistungen weiterhin eine wichtige Stellung eingeräumt, gleichzeitig wird vor der Gefahr der Einengung individueller Freiheiten durch ein Übermaß an staatlicher Bürokratie gewarnt. Konkret schlägt die Partei eine Aufgabenteilung vor, die sich an den Grundsätzen des Gemeinwohls, der Leistungsqualität sowie der Kosteneffizienz orientiert (SPÖ 1998: 21f.):

„Ob Leistungen gemein- oder privatwirtschaftlich erbracht werden, ist danach zu entscheiden, wer (...) die Anforderungen in Bezug auf Qualität, Effizienz und Wirtschaftlichkeit am besten erfüllt, wobei vor allem auch der gesamtgesellschaftliche Nutzen berücksichtigt werden muss" (SPÖ 1998: 21).

Über die Zeit hinweg konstante Zielsetzungen sind Bekämpfung der Arbeitslosigkeit, Investitionstätigungen in die Infrastruktur sowie das Eintreten für eine Einkommensverteilung. Neu ist hingegen die Vorstellung mittels institutioneller Rahmenbedingungen freie Wettbewerbsbedingungen $\mathrm{zu}$ garantieren. Von besonderer Bedeutung ist hierbei ein Wirtschafts- und Kartellrecht zur Verhinderung von Machtkonzentrationen (SPÖ 1998: 9).

\subsubsection{Menschenbild}

Das Menschenbild im 78er-Programm orientiert sich am Leitprinzip einer solidarischen Gesellschaft, in der nicht das eigeninteressierte, sondern ein in die Gemeinschaft eingebettetes Individuum im Zentrum der Betrachtung steht. Die SPÖ wendet sich gegen die Vorstellung einer ausschließlich auf individueller Leistungserbringung beruhenden Verbesserung der eigenen Lebenssituation. Stattdessen wird die persönliche Selbstverwirklichung von einem funktionierenden Gesellschaftssystem abhängig gemacht:

„Wir Sozialisten wissen, dass nur eine Verbesserung der Lebensverhältnisse aller Mitglieder einer Gesellschaft auch die Verbesserung der Lebensverhältnisse des einzelnen zu sichern vermag" (SPÖ 1978: 14).

Angesichts des ständigen Leistungsdrangs und des hohen Grads an Fremdbestimmtheit fordern die Sozialisten eine Humanisierung der Arbeitswelt. Menschliche Arbeit soll demnach nicht vorrangig Werkzeug für 
bloße Profit- und Einkommensgenerierung sein, sondern einen darüber hinaus gehenden sinnstiftenden Charakter einnehmen (SPÖ 1978: 11, 37).

In der Schrift von 1998 wird an die Vorstellung der sozialen Verantwortlichkeit des Einzelnen für die Gemeinschaft angeknüpft. So ist es nach wie vor das Bestreben der Partei eine Gesellschaftsordnung zu errichten, die sich an solidarischen Prinzipien orientiert und zugleich die freie Entfaltung der menschlichen Persönlichkeit garantiert. Im Unterschied zur Vorgängerprogrammatik wird jedoch die Abhängigkeit des individuellen Wohlergehens von den gesamtgesellschaftlichen Verhältnissen fallen gelassen (SPÖ 1998: 5f.):

„Wir stehen für eine Politik, die die Menschen in die Lage versetzt, ihr Leben selbstbestimmt und mündig $z u$ gestalten, und wollen gesellschaftliche Bedingungen schaffen, die diesem Prinzip entsprechen" (SPÖ 1998: 6).

Aufgabe des Staats ist es, die BürgerInnen vor potenziell negativen Auswirkungen der Marktkräfte zu schützen (SPÖ 1998: 7), zugleich muss staatliches Handeln darauf gerichtet sein, Autonomie und Eigenverantwortung des Einzelnen zu steigern (ebd.: 20).

Auch bei der Ausgestaltung der menschlichen Arbeitswelt ist ein Bedeutungswandel feststellbar. So wird der Primat einer sinnstiftenden Arbeit aus dem 78er-Programm zugunsten einer bloßen Partizipation im Arbeitswesen ersetzt. In diesem Sinn steht nun die Schaffung von lebensunterhaltsichernder Erwerbsarbeit im Vordergrund (ebd.: 3, 7).

\subsubsection{Wohlfahrtsstaatliches Engagement}

Die SPÖ hebt im Grundsatzprogramm von 1978 ihren maßgeblichen Anteil am Aufbau eines umfassenden wohlfahrtsstaatlichen Apparats hervor. Diesem kommt nicht nur eine existenzsichernde Funktion zu, sondern er hat darüber hinaus die stetige Verbesserung der Lebensverhältnisse (z. B. Recht auf Arbeit, Recht auf eine humane Umwelt) sowie die aktive Teilnahme aller BürgerInnen am Gemeinschaftsleben $\mathrm{zu}$ gewährleisten:

„Das System der sozialen Sicherheit muss so ausgebaut werden, dass jedem Österreicher die Freiheit von Not sowie seine Teilnahme am gesellschaftlichen und kulturellen Leben garantiert ist" (SPÖ 1978: 38).

Soziale Sicherheit wird dabei als eine vorrangig staatliche Aufgabe verstanden, die nur in geringem Ausmaß durch private Vorsorgeleistungen ergänzt werden kann (SPÖ 1978: 37).
Die Sozialisten traten, ausgehend von ihrem Befund eines beträchtlichen Maßes an Einkommensungleichheit in der österreichischen Gesellschaft, für verteilungspolitische Maßnahmen zugunsten niedriger Einkommensschichten ein. Die Ungleichheit sollte dabei v. a. durch eine solidarische Lohnpolitik sowie eine entsprechende Steuerpolitik verringert werden (ebd.: 26).

Auch in der Programmatik von 1998 werden sozialstaatliche Leistungen als Basis für sozialen Frieden und individuelle Entfaltung angesehen. Wohlfahrtsstaatliche Maßnahmen inkludieren dabei sowohl das Ziel der Vollbeschäftigung als auch ein solidarisches Sicherungsnetz, das den Bedürftigen als Existenzsicherung dient:

„Sicherheit und Wohlfahrt verstehen wir dabei in einem sehr umfassenden Sinne: Das Ziel der Vollbeschäftigung gehört ebenso dazu wie solidarische Sicherungssysteme für Alter, Krankheit, Behinderung, Unfall und Arbeitslosigkeit sowie ein sozial gerechtes System von Transferleistungen" (SPÖ 1998: 12).

Die im Vorgängerprogramm ausgegebene Zielvorstellung der gesellschaftlichen Partizipation aller BürgerInnen wird hingegen nicht mehr thematisiert. Vielmehr findet eine Reduktion der Zielgruppe statt, wenn es heißt, dass „tatsächlich jenen geholfen wird, die in Notlage geraten sind“ (ebd.: 1998: 12). Überdies wird von den Menschen Eigenverantwortung in Form privater Vorsorgeleistungen gefordert (SPÖ 1998: 12).

Bei der Verteilung von Einkommen wird das Leistungsprinzip als Legitimation für Einkommensunterschiede herangezogen, wenngleich die Ungleichverteilung nicht zulasten einer beständigen Teilnahme aller am gesellschaftlichen Leben gehen darf (ebd.: 9).

\subsection{Interpretation der Parteiprogramme der FPÖ}

Das Freiheitliche Manifest zur Gesellschaftspolitik (Beschluss: 05./06.1973 in Linz) bildete den Abschluss einer seit Ende der 196oer-Jahre andauernden programmatischen Umgestaltung, die ursprünglich von Parteiobmann Peter zur stärkeren Betonung liberaler Elemente initiiert wurde. Im Manifest werden neben der zentralen Stellung des Individuums auch dessen Verbindung zur organisch gewachsenen Gemeinschaft betont (FPÖ 1973: 13). Das im Oktober 1997 ebenfalls in Linz verabschiedete Programm wurde nach der Abspaltung des BZÖ am 4. April 2005 auf dem Bundesparteitag in Salzburg, auf dem auch Strache zum neuen 
Parteiobmann gewählt wurde, geringfügig modifiziert. Inhaltlich wird v. a. auf (Handlungs-)Freiheit und ein schlankes Staatswesen großer Wert gelegt.

\subsubsection{Marktbild}

Im Programm von 1973 ist eine grundsätzlich positive Sichtweise in Bezug auf das kapitalistische Wirtschaftssystem erkennbar. So gibt die Partei ein Bekenntnis zur sozialen Marktwirtschaft $\mathrm{ab}$, in der insbesondere die Freiheit des Unternehmertums als zentrale Voraussetzung für wirtschaftlichen Erfolg angesehen wird:

„Wir Freiheitlichen stehen auf dem Boden der sozialen Marktwirtschaft. Ein genügend großer Spielraum des eigenverantwortlichen Unternehmers ist eine wichtige Voraussetzung für eine leistungsfähige Wirtschaft, die von der gemeinsamen Arbeit aller getragen wird" (FPÖ 1973: 24).

In diesem Zusammenhang streicht die Partei auch die Überlegenheit der sozialen Marktwirtschaft gegenüber einem zentral gelenkten Wirtschaftssystem heraus, dessen wirtschaftlicher Entwicklungsstand verhältnismäßig niedriger eingeschätzt wird (FPÖ 1973: 55). Die Freiheitlichen sehen jedoch die marktwirtschaftliche Stabilität aus sich heraus nicht gegeben und befürworten in weiterer Folge staatliche Regulierungsund Steuerungsversuche, wobei diese stets im Sinne des Marktes zu erfolgen haben (ebd.: 25).

Im Programm von 2005 versucht sich die FPÖ mit der Formel der fairen Marktwirtschaft sowohl von einem schrankenlosen Kapitalismus als auch vom gescheiterten Realsozialismus abzugrenzen. Im Rahmen der (fairen) Marktwirtschaft ermöglichen individuelle Freiheit und unternehmerische Leistungsstärke eine erfolgreiche Wirtschafts- und Gesellschaftsentwicklung:

„Faire Marktwirtschaft sichert durch freien Wettbewerb in sozialer Verantwortung eine dynamische Wirtschaftsentwicklung. (...) Faire Marktwirtschaft fördert die Leistungsbereitschaft und weckt die schöpferischen Kräfte" (FPÖ 2005: 18).

Im Unterschied zur Schrift von 1973 ist in jener von 2005 nun gerade die Abwesenheit von staatlichen Eingriffen notwendige Voraussetzung für ein wettbewerbsund leistungsfähiges Wirtschaftssystem. Überhaupt ist das gesamte der Marktwirtschaft gewidmete Kapitel 10 in der Programmatik von einem staatsskeptischen Denken durchzogen. Akzeptiert werden institutionelle Ordnungsbedingungen, v. a. im Hinblick auf die Verhinderung monopolistischer Strukturen. Auch soll der von politischen Parteien und Verbänden ausgehende Einfluss auf einzelne Wirtschaftsbereiche mittels Privatisierungen durchbrochen werden (FPÖ 2005: 18f.).

\subsubsection{Wirtschaftspolitik}

In der Programmatik von 1973 zählt die Stabilisierung des marktwirtschaftlichen Systems sowie im Besonderen die Wahrung geordneter Wettbewerbsverhältnisse zu den vorrangigen Arbeitsfeldern der Wirtschaftspolitik. Die staatlichen Instrumentarien sollen sich dabei weniger an ideologischen Standpunkten denn an ihrer Zweckmäßigkeit orientieren. In diesem Sinne sieht die FPÖ auch die Möglichkeit eines planenden Eingriffes in den laufenden Wirtschaftsprozess, insofern dies der Sicherung der wirtschaftlichen Stabilität oder der Erfüllung gemeinschaftlicher Aufgaben (z. B. Sozialpolitik, Daseinsvorsorge) dient (FPÖ 1973: 25, 51f.):

„Der moderne Staat greift tief in den Wirtschaftsprozess ein. Wir bejahen diese Eingriffe soweit sie darauf abzielen, eine funktionierende soziale Marktwirtschaft zu sichern. Hingegen lehnen wir jede staatliche Wirtschaftspolitik ab, die eine Verstaatlichung der Wirtschaft oder ihre Umwandlung in eine staatlich gelenkte Planwirtschaft bezwecken" (FPÖ 1973: 25).

Obwohl Verstaatlichungen als allgemeiner Grundsatz entschieden abgelehnt werden, soll die betriebliche Leistungserstellung dem Grundsatz der Gleichberechtigung zwischen verstaatlichten und privaten Unternehmen unterliegen (FPÖ 1973: 29).

Rund dreißig Jahre später haben sich die Ansichten der FPÖ evident in Richtung einer staatskritischen Haltung verschoben. Nunmehr steht, neben der Stärkung der Wettbewerbsfähigkeit kleiner und mittlerer Unternehmen, v. a. die Reduzierung auf einen möglichst schlanken Staatskorpus im Mittelpunkt freiheitlicher Wirtschaftspolitik. Vor allen Dingen darf der Staat nicht als Unternehmer auftreten:

„Der Staat sollte sich jeder erwerbswirtschaftlichen und unternehmerischen Betätigung enthalten müssen, die derzeit lediglich zur Verzerrung der Wettbewerbsverhältnisse auf dem Markt zu Lasten der privaten Mitbewerber (...) führt" (FPÖ 2005: 13).

Ein Staatsaufgabenkatalog soll die „Konzentration auf die ureigensten Staatsaufgaben“ (ebd.: 12) ermöglichen, zu denen die Wahrung der inneren und äußeren Sicherheit, die Durchsetzung einer Gesetzgebung sowie die Sicherstellung der Grundversorgung zählen (FPÖ 2005: 12). Damit einhergehend ist auch ein weitläufiges Deregulierungs- und Privatisierungsbestreben, 
um den Einfluss politischer Parteien auf die Wirtschaft zu vermindern. Ein schlanker Staatskorpus ermöglicht in weiterer Folge eine Reduzierung der Abgabenquote für Unternehmen (Wettbewerbssteigerung) und MitarbeiterInnen (ebd.: 18ff.).

\subsubsection{Menschenbild}

Das Menschenbild in der Schrift von 1973 orientiert sich am Grundsatz einer freiheitlichen Lebensordnung, die den Fokus auf die Selbstbestimmung der Individuen legt (FPÖ 1973: 9, 55). Als Konsequenz der Sichtweise einer von gemeinschaftlichen Verhältnissen möglichst unabhängigen persönlichen Entwicklung wird das materialistische Gesellschaftsverständnis kritisiert:

„Die linken Utopisten betrachten den Menschen als

Produkt seiner Umwelt und der Produktionsverhältnisse.

Damit verneinen sie die Freiheit des Menschen, denn der

Mensch als Produkt der Verhältnisse hätte keine Freiheit"

(FPÖ 1973: 6).

Die Entfaltung der Persönlichkeit ist in erster Linie auf die Abwendung staatlicher Bevormundung zurückzuführen. Darüber hinaus kommt bei der individuellen Entwicklung auch der Familie eine tragende Rolle $\mathrm{zu}$, da sie im freiheitlichen Verständnis die wichtigste Form der Gemeinschaft darstellt (FPÖ 1973: 9, 14).

Arbeit wird für das menschliche Leben als zentral eingestuft. Eine sinnerfüllte Tätigkeit bildet zusammen mit einem leistungsbewussten Denken die Basis der Arbeitswelt (ebd.: 24).

Individuelle Freiheit und Eigenverantwortung sind auch in der Programmatik von 2005 die zentralen Postulate der FPÖ. In diesem Kontext wird auf die Gegensätzlichkeit eines auf staatlichen Zwangsmaßnahmen beruhenden Gesellschaftssystems im Vergleich zu einer freiheitlichen Ordnung hingewiesen. Der Freiheitsbegriff ist wie im Vorgängerprogramm - durch die Abwesenheit von Zwang - primär negativ definiert:

"Staatliche Umerziehung, Bevormundung und Gängelungen sind im Grunde gegen die Menschenwürde gerichtet und verneinen die Verschiedenartigkeit aller Menschen. Zwangsbeglückungen oder gar totalitäre Umformungen zu einem neuen Menschen nach vorbestimmten Normen und ideologischen Konzepten sind mit der Achtung der Menschenwürde unvereinbar" (FPÖ 2005: 3).

Eine weitere Parallele zur Schrift von 1973 findet sich in der sozialen Eingebundenheit des Einzelnen in die Gemeinschaft, wobei wiederum der Familie die gewichtigste Position zukommt (FPÖ 2005: 1).
In der Programmatik von 2005 finden sich wenig konkrete Aussagen zur Ausgestaltung der menschlichen Arbeitswelt, da hauptsächlich Maßnahmen zur Förderung der Unternehmen vorgeschlagen werden. Die Angaben beschränken sich auf die Formulierung einer „verantwortlichen Partnerschaft zwischen Arbeitgebern und Arbeitnehmern" (FPÖ 2005: 19) zum Zwecke der Steigerung der Leistungsbereitschaft in der Belegschaft.

\subsubsection{Wohlfahrtsstaatliches Engagement}

Sozialstaatliche Elemente haben in der Programmatik von 1973 die Aufgabe, den Menschen eine Absicherung gegen die Risiken einer auf Freiheit beruhenden Gesellschaftsordnung zu bieten (FPÖ 1973: 12). Grundsätzlich ist es Angelegenheit des Staates allen Menschen, die nicht mehr befähigt sind für ihren eigenen Lebensunterhalt aufzukommen, ein Mindesteinkommen (Grundversorgung) zu garantieren:

„Schutz vor äußerer Gewalt (...) war schon immer die ureigenste Aufgabe jedes Staates. Darüber hinaus braucht der Mensch auch soziale Sicherheit. Sie muss in der extrem arbeitsteilig organisierten und technisierten Zivilisation als zusätzliche gesellschaftspolitische Aufgabe des Staates wahrgenommen werden" (FPÖ 1973: 37).

Zugleich wird auf die Gefahren umfassender Versorgungsleistungen hingewiesen, die eine völlige Abhängigkeit der BürgerInnen zur Folge haben. Zur Vermeidung solcher Abhängigkeitsverhältnisse sind die Menschen dazu angehalten auch selbst für ihre eigene Sicherheit Vorsorge zu leisten (FPÖ 1973: 57).

Die FPÖ sieht im personellen Einkommen einen wesentlichen Faktor für Leistungsanreize. In diesem Sinne strebt sie, für eine Steigerung der individuellen Leistungsbereitschaft, anstelle einer stärkeren Gleichverteilung eine bewusste Einkommensdifferenzierung an. Daneben soll aber auch eine sinnerfüllte Arbeit einen wichtigen Leistungsanreiz darstellen (ebd.: 25).

In der Fassung von 2005 wird die Gesellschaft als „solidarische Risikogemeinschaft“ (FPÖ 2005: 21) bezeichnet, in der die Menschen die sozialen Aufgaben in erster Linie eigenverantwortlich oder in kleinen Kreisen (z. B. Familie) wahrzunehmen haben. Der Staat hat als letzte Instanz die Verantwortung für die soziale Wohlfahrt zu übernehmen, dabei muss er neben der Verhinderung existenzieller Notlagen auch die Sicherung von Mindestlebensbedingungen garantieren (FPÖ 2005: 21):

„Der Staat als letztverantwortliche Organisationsform der Risikogemeinschaft soll hierbei lediglich eine 
Grundversorgung gewährleisten, die die eigenverantwortliche Vorsorge ergänzt und vervollständigt" (FPÖ 2005: 22).

Im Unterschied zum 73er-Programm wird nun neben der Gefahr von Abhängigkeitsverhältnissen auch jene des Sozialschmarotzertums infolge umfassender Versorgungsleistungen problematisiert. Notwendig ist daher eine effektive Bekämpfung von Sozialleistungsmissbrauch (FPÖ 2005: 22).

\subsection{Interpretation der Parteiprogramme der Grünen}

Die Grüne Alternative ging 1986 aus einer Vereinigung zwischen den Vereinten Grünen Österreichs und der Alternativen Liste Österreichs hervor. Bei den Nationalratswahlen im selben Jahr schaffte die Partei mit der Spitzenkandidatin Meissner-Blau zum ersten Mal den Einzug ins Parlament. Im 1986 ausgearbeiteten Kurzprogramm stellt die Partei „das vorläufige Ergebnis unserer Zusammenarbeit vor" (Grüne 1986: 3), da sie aufgrund des vorgezogenen Wahltermins mitten in der Programmarbeit überrascht wurden (Grüne 1986: 3). Inhaltlich ist die Programmatik stark am Umweltschutz ausgerichtet. Dem beim 20. Bundeskongress von Linz am 07./o8. Juli 2001 beschlossenen Grundsatzprogramm gingen mehrere Diskussionen auf Bundestagungen voraus. Im Vorwort des Programms sehen sich die Grünen als „Alternative im Parteisystem“ (Grüne 2001: ii), wenngleich der Text von stärkerem Pragmatismus durchzogen ist.

\subsubsection{Marktbild}

Im Leitlinienprogramm von 1986 ist ein unter ökologischen Gesichtspunkten negatives Bild von der kapitalistischen Marktwirtschaft ersichtlich. Dabei werden v. a. die - infolge der unkontrollierten Wirtschaftsentwicklung - fortschreitende Ausbeutung und Zerstörung der Umwelt sowie die damit verbundenen negativen Konsequenzen für die Menschen, etwa in Form der Entziehung der Lebensgrundlagen, thematisiert:

„Umweltzerstörung, Ausbeutung wichtiger Rohstoffe und Wettrüsten gehen unvermindert weiter. Die Zerstörung der Natur hat in den letzten Jahren katastrophale Ausmaße angenommen. Die Natur leidet an wildem Wachstumszwang einer zerstörerischen Industriegesellschaft, die Menschen leiden an der zerstörten Natur" (Grüne 1986: 2).
Die Kritik an der Umweltzerstörung beschränkt sich keinesfalls auf den privaten Unternehmensbereich. Vielmehr vertritt die Partei den Standpunkt der prinzipiellen Unvereinbarkeit zwischen einer auf Profitorientierung und Massenproduktion ausgerichteten kapitalistischen Wirtschaftsordnung und einer ökologischen Verträglichkeit. Dieser Zustand kann nur durch einen grundlegenden Umbau des gesamten Wirtschaftssystems überwunden werden (Grüne 1986: 6f.).

Die aktuelle Programmatik folgt einer differenzierten Betrachtung, da sowohl Stärken als auch Schwächen der kapitalistischen Marktwirtschaft behandelt werden. Auf der einen Seite wird in Übereinstimmung mit dem 86er-Programm die Ausbeutung natürlicher und menschlicher Ressourcen kritisiert. Zudem warnen die Grünen vor der zunehmenden Tendenz der Ökonomisierung sozialer Lebensbereiche (Grüne 2001: 18). Auf der anderen Seite verweisen sie auf die effiziente Koordinationsfähigkeit der Marktkräfte in wirtschaftlichen Prozessen:

„Die Grünen erkennen an, dass der Markt das effizienteste bisher bekannte Steuerungsinstrument für wirtschaftliche Aktivitäten ist; nicht weniger, aber auch nicht mehr" (Grüne 2001: 18).

Da der Markt per se jedoch keine gesellschaftlichen Ziele hervorbringt, soll er dem Primat der Politik untergeordnet sein. Wenn durch staatliches Handeln dort regulierend eingegriffen wird, wo es $\mathrm{zu}$ unerwünschten Ergebnissen für Mensch und Umwelt führt, kann der Marktmechanismus für ein soziales und ökologisches Wirtschaften nutzbar gemacht werden (Grüne 2001: 11, 18).

\subsubsection{Wirtschaftspolitik}

Ausgehend vom Befund einer umwelt- und menschenschädlichen Wirtschaftsordnung streben die Grünen in ihrem Kurzprogramm eine ökologische und soziale Systemtransformation an:

„Dieses Wirtschaftssystem ist menschenfeindlich. Kurzfristig müssen wir seine schlimmsten Folgen mindern. Langfristig müssen wir es umbauen" (Grüne 1986: 6).

Die ökologische Sanierung der Wirtschaft soll bei den verstaatlichten Unternehmen beginnen - anstelle schädlicher Massenproduktionen (z. B. Aluminium, Stahl) ist dabei verstärkt auf umweltfreundliche Finalerzeugnisse umzusteigen. Privatisierungen staatlicher Betriebe werden abgelehnt (Grüne 1986: 7). Darüber hinaus benötigt die ökologische Sanierung eine dem 
Credo des Umweltschutzes verpflichtete Wirtschaftspolitik. Hierzu zählen u. a. das Herabsetzen von Grenzwerten für den Schadstoffausstoß in Industrie (z. B. Produktionsumstrukturierung) und Verkehr (z. B. autofreie Sonntage) sowie eine Besteuerung ökologisch externer Effekte nach dem Verursacherprinzip (ebd.: 3, 8). Ein weiteres programmatisches Kernanliegen ist die Verringerung der nationalen Einkommensunterschiede hinsichtlich Arbeit bei einer gleichzeitigen steuerlichen Begünstigung von Arbeitszu Nichtarbeitseinkommen (ebd.: $7 \mathrm{f}$.).

In der Programmatik von 2001 wird entsprechend dem vermittelten Marktbild ein wirtschaftspolitischer Zugang verfolgt, der neben der ökologischen Verträglichkeit auch die Notwendigkeit der ökonomischen Funktionalität miteinbezieht (Grüne 2001: 13). Der Handlungsspielraum des Staates sieht dabei neben dem Setzen von Rahmenbedingungen auch die Möglichkeit von konkreten Steuerungen im Hinblick auf eine soziale und nachhaltige Wirtschaftsordnung vor. Einer vorzugsweise auf die Unternehmerseite ausgerichteten Wirtschaftspolitik wird hingegen eine Absage erteilt:

„Wirtschaftspolitik kann sich daher aus Grüner Sicht nicht in der Erhöhung der Vorteile von Unternehmen erschöpfen. (...) Für die Politik bedeutet dies einen klaren Auftrag, dem betrieblichen Wirtschaften einen Rahmen zu setzen. Dieser hat die Kosten und Nutzen im betriebs- und branchenübergreifenden, gesamtwirtschaftlichen Zusammenhang zu berücksichtigen und unsere natürlichen Lebensgrundlagen zu sichern" (Grüne 2001: 19f.).

Obgleich die staatliche Aufgabe der Bereitstellung und Verteilung öffentlicher Güter und Dienstleistungen (Daseinsvorsorge) unbestritten ist, werden Privatisierungskonzepte nicht mehr pauschal verworfen. Diese sollen im Hinblick auf gesellschaftliche, ökologische und wirtschaftliche Auswirkungen überprüft werden (Grüne 2001: 18, 21). Die Steuerpolitik zielt analog zur Vorgängerprogrammatik sowohl auf eine stärkere Besteuerung von Energieverbrauch als auch auf eine Entlastung von Arbeitseinkommen ab (ebd.: 22).

\subsubsection{Menschenbild}

Das Kurzprogramm von 1986 enthält noch keine grundlegend ausgearbeiteten Wertevorstellungen über das menschliche Zusammenleben. Der Fokus liegt auf der Aussage der ökologischen Einbettung des Menschen in ein übergeordnetes System. Aufgrund der zentralen Wechselwirkungen zwischen Mensch und Umwelt sehen die Grünen den Fortbestand der menschlichen Spezies als gefährdet an. Weil der Mensch durch Umweltverschmutzung und Ressourcenverbrauch das Ökosystem, in das er eingebettet ist, zusehends zerstört, vernichtet er zugleich seinen eigenen Lebensraum (Grüne 1986: 2).

Die menschliche Arbeitswelt wird v. a. vor dem Hintergrund einer zunehmenden Technisierung (z. B. Mikroelektronik) und der damit verbundenen Rationalisierung von Arbeitsplätzen betrachtet. Im Sinne einer „menschengerechte[n] Technologiepolitik“ (Grüne 1986: 8) muss vor der Einführung neuer Technik ihre tätigkeitsunterstützende Funktion nachgewiesen sein (Grüne 1986: 8).

Fünfzehn Jahre später liegt dem Grundsatzprogramm ein detailliertes Menschenbild vor, das u. a. auf den Prinzipien des sozialen Zusammenhalts (Solidarität) und der Bewahrung der natürlichen Lebensgrundlagen (Ökologie) beruht. Im Rahmen der ökologischen Einbettung wird auf die Bedeutung einer intakten Umwelt sowie einer gerechten Ressourcenverteilung hingewiesen. Die soziale Einbettung des Menschen findet in Form der allgemeinen Pflicht zur gegenseitigen Hilfe im Gemeinschaftswesen Ausdruck und ist dem Recht auf Selbstbestimmung zur Seite gestellt. Dabei wird die Verwirklichung der individuellen Freiheit davon abhängig gemacht, inwiefern es in einer Gesellschaft gelingt, soziale Rahmenbedingungen und einen ökologischen Ausgleich der Ressourcen sicherzustellen:

„Selbstbestimmung und Solidarität stehen potentiell in einem Spannungsverhältnis, solange nicht berücksichtigt wird, dass erst eine solidarische Verteilung der Ressourcen allen Menschen ein selbstbestimmtes Leben ermöglicht“ (Grüne 2001: 7).

Die Grünen betrachten Erwerbsarbeit nicht ausschließlich aus ökonomischem Kalkül (Existenzsicherung, Wirtschaftswachstum). Eine sinnerfüllte Arbeit ist darüber hinaus Quelle von Selbstverwirklichung und Anerkennung. Daneben weisen sie auf die - mit der Zunahme atypischer Beschäftigungsverhältnisse verbundene - gesellschaftliche Segmentierung hin (Grüne 2001: 31f.).

\subsubsection{Wohlfahrtsstaatliches Engagement}

Die Grünen proklamieren in ihrem Kurzprogramm eine Opferpolitik, bei der nicht individuelles, sondern gesellschaftliches Versagen als Gründe für Arbeitslosigkeit, Armut und soziale Ausgrenzung angeführt werden. Vor diesem Hintergrund sieht die Partei ein 
Tabelle 2: Übersicht programmatische Kerninhalte - Marktbild

\begin{tabular}{|c|c|c|}
\hline \multirow{8}{*}{ 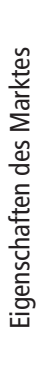 } & ÖVP 1972 & Förderung von Wohlstand; Lösen von Gemeinschaftsaufgaben \\
\hline & ÖVP 2015 & Anreize für Wachstum, Wohlstand und wirtschaftliche Nachhaltigkeit \\
\hline & SPÖ 1978 & Schädigung von Mensch und Umwelt; Systeminstabilität \\
\hline & SPÖ 1998 & Förderung von Wohlstand, aber fehlende Verteilungsgerechtigkeit \\
\hline & FPÖ 1973 & Freiheits- und fortschrittsfördernd bei adäquater staatlicher Regulierung \\
\hline & FPÖ 2005 & Förderung Leistungsbereitschaft und dynamische Wirtschaftsentwicklung \\
\hline & Grüne 1986 & Ausbeutung und Zerstörung der Umwelt \\
\hline & Grüne 2001 & Ausbeutung und Zerstörung der Umwelt; effizientestes bekanntes System \\
\hline
\end{tabular}

Quelle: Eigene Darstellung

umfassendes System der sozialen Sicherheit vor, bei dem analog zur Idee des bedingungslosen Grundeinkommens für alle BürgerInnen eine erwerbsunabhängige soziale Grundsicherung zur Verfügung stehen soll. Darüber hinaus sind von staatlicher Seite auch nichtmonetäre Leistungen in Form von Pflege- und Betreuungseinrichtungen zur Verfügung zu stellen (Grüne 1986: 6).

Die Grünen sehen in Anbetracht ungleicher Einkommens- und Vermögensverhältnisse auch die Notwendigkeit einer gerechteren Verteilung des Volksvermögens:

„Wenn Manager, Primarärzte und Spitzenpolitiker jährliche Einkommen beziehen, die für die meisten Menschen nicht einmal im Laufe ihres ganzen Lebens $z u$ erzielen sind, dann reden jene, die diesen Zustand als untragbar bezeichnen, gewiss nicht der Gleichmacherei das Wort" (Grüne 1986: 7).

Auch im Programm von 2001 wird ein umfassendes soziales Sicherungssystem angestrebt, da Individuen trotz Erwerbstätigkeit oftmals von Armut betroffen sind. Das System setzt sich wiederum aus einer monetären Grundsicherung und sozialstaatlichen Rahmenbedingungen (z. B. Kinderbetreuungseinrichtungen, Gesundheitswesen) zusammen (Grüne 2001: 37, 48f.). Mit dem Modell der bedarfsorientierten Grundsicherung sollen alle BürgerInnen ein Mindesteinkommen beziehen, das neben der Existenzsicherung auch die Teilnahme am gesellschaftlichen Leben ermöglicht:

„Die Grünen wollen Existenzsicherung vom konkreten Erwerbsarbeitsverhältnis entkoppeln: Alle Menschen haben das Recht auf eine menschenwürdige Existenz. Dazu gehört eine finanzielle Grundsicherung ebenso wie die Chance, die eigenen Fähigkeiten umfassend zu entwickeln. Die Grüne Grundsicherung will Teilhabe an der Gesellschaft fördern"(Grüne 2001: 30).

Inhaltliche Kontinuität besteht auch in der Auffassung einer ausgewogeneren Verteilung, wobei die
Schwerpunkte in den Bereichen der Einkommensverteilung zwischen Löhnen und Profiten sowie zwischen Männern und Frauen gesetzt werden (ebd.: 36).

\section{Schlussfolgerungen und Diskussion}

In diesem Beitrag wurden Grundsatzprogramme der einflussreichsten österreichischen Parteien hinsichtlich der Übernahme von neoliberalen Standpunkten analysiert. Ausgehend von den ermittelten Ergebnissen kann eine parteiübergreifende Annäherung an neoliberale Positionen konstatiert werden, wenngleich die Intensität der Annäherung zwischen den einzelnen Fraktionen variiert. In Bezug auf die analysierten Dimensionen konnten dabei folgende Veränderungen festgestellt werden (Tabellen 2-5):

Standen einige Parteien in ihren frühen Schriften der Marktwirtschaft sehr kritisch gegenüber (SPÖ, Grüne) oder betonten zumindest die Notwendigkeit einer politischen Steuerung (FPÖ) bzw. Regelung (ÖVP), so lässt sich in allen aktuellen Programmen eine parteiübergreifende Bewegung hin zu einer marktfreundlichen Position erkennen. Der Markt wird dabei als (effizientestes) Allokationsinstrument für ökonomische Aktivitäten wahrgenommen, wenngleich bei SPÖ und Grünen weiterhin gewisse Einschränkungen vorhanden sind (Tabelle 2).

Verbunden mit dieser optimistischeren Ausrichtung änderte sich auch die Form der Wirtschaftspolitik. Waren Systemtransformation (SPÖ, Grüne) bzw. -erhaltung (ÖVP, FPÖ) wesentliche Ziele in den Programmen aus der Golden-Age-Phase des Kapitalismus, ist nun die Sicherung der Wettbewerbsfähigkeit eine Gemeinsamkeit in der wirtschaftspolitischen Ausrichtung aller Parteien. Entsprechend wurden keynesianisch angelehnte Regulierungs- und Interventionskonzepte zur Steuerung der Marktwirtschaft zugunsten einer auf die Ausgestaltung und Sicherung 
Tabelle 3: Übersicht programmatische Kerninhalte - Wirtschaftspolitik

\begin{tabular}{|c|c|c|c|}
\hline \multirow{9}{*}{ 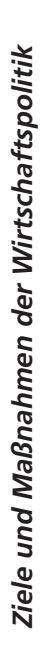 } & & Ziele & Maßnahmen \\
\hline & ÖVP 1972 & $\begin{array}{l}\text { Geldwertstabilität, Vollbeschäftigung, } \\
\text { Wachstum }\end{array}$ & Wirtschaftsnahe Steuer-, Konjunktur- und Investitionspolitik \\
\hline & ÖVP 2015 & $\begin{array}{l}\text { Stärkung der internationalen Wettbewerbs- } \\
\text { fähigkeit, schlanker Staat }\end{array}$ & Unternehmensfreundliches Steuersystem, Privatisierung, Flexibilisierung \\
\hline & SPÖ 1978 & $\begin{array}{l}\text { Transformation zu einem "demokratischen } \\
\text { Sozialismus" }\end{array}$ & $\begin{array}{l}\text { Planender demokratischer Prozess, staatliche Nachfragestützung, } \\
\text { Entlastung kleiner Einkommen }\end{array}$ \\
\hline & SPÖ 1998 & Vollbeschäftigung, Wettbewerbssicherung & Staatlicher Ordnungsrahmen, Investitionspolitik \\
\hline & FPÖ 1973 & $\begin{array}{l}\text { Systemstabilisierung, geordnete Wettbew- } \\
\text { erbsbedingungen }\end{array}$ & Steuernde Eingriffe des Staates \\
\hline & FPÖ 2005 & $\begin{array}{l}\text { Wettbewerbsstärkung kleiner und mittlerer } \\
\text { Unternehmen, schlanker Staat }\end{array}$ & $\begin{array}{l}\text { Staatsaufgabenkatalog, Privatisierung, Deregulierung, } \\
\text { leistungsfreundliches Steuersystem }\end{array}$ \\
\hline & Grüne 1986 & Ökologische Transformation & Umweltfreundliche Produktion, ökologisches Steuersystem \\
\hline & Grüne 2001 & $\begin{array}{l}\text { Ökologische Verträglichkeit, wirtschaftliche } \\
\text { Effizienz }\end{array}$ & Rahmenbedingungen und Eingriffe, ökologisches Steuersystem \\
\hline
\end{tabular}

Quelle: Eigene Darstellung

Tabelle 4: Übersicht programmatische Kerninhalte - Menschenbild

\begin{tabular}{llll}
\hline & & Grundwerte & Arbeitsverständnis \\
\cline { 2 - 4 } & ÖVP 1972 & Solidarität, Subsidiarität & Leistungserbringung, Selbstverwirklichung \\
& ÖVP 2015 & Individuelle Freiheit, Eigenverantwortung & Leistungserbringung, Anreizorientierung \\
& SPÖ 1978 & Entfaltung im Rahmen einer Gemeinschaft & Humanisierung der Arbeitswelt \\
Grüne 1986 & Ökologische und soziale Einbettung & Existenzsicherung \\
Grüne 2001 & Ökologische und soziale Einbettung & Leistungserbringung, Lebenserfüllung \\
& FPÖ 1973 & Individuelle Freiheit, Eigenverantwortung, Familie & Leistungserbringung, Anreizorientierung \\
\end{tabular}

Quelle: Eigene Darstellung

Tabelle 5: Übersicht programmatische Kerninhalte - Wohlfahrtsstaat

\begin{tabular}{lll}
\hline & ÖVP 1972 & Versorgung und Verbesserung Lebensverhältnisse durch Staat, Eigeninitiative \\
ÖVP 2015 & Zeitlich begrenzte Grundversorgung zur Stärkung der Eigenverantwortung \\
Umfassende Sicherung und Verbesserung Lebensverhältnisse durch Staat \\
SPÖ 1978 & Staatliche Sicherung für Berechtigte, Eigeninitiative \\
Grüne 1986 & Staatliche Sicherung für Berechtigte, Eigeninitiative \\
GPÖn 2001 & Eetonung Eigenverantwortung, Staat als letztverantwortliche Instanz \\
& FPÖ 2005 & Erwerbsunabhängiges Grundeinkommen, sozialstaatliche Einrichtungen \\
\hline
\end{tabular}

Quelle: Eigene Darstellung

einer funktionierenden Marktwirtschaft ausgerichteten Wirtschaftspolitik ersetzt (Tabelle 3).

Beim Menschenbild gibt es sowohl Konstanz als auch Veränderung. So konnte nur bei zwei Parteien eine merkbare Änderung ihrer Grundwerte - in Richtung der Stärkung der individuellen Freiheit (ÖVP,
SPÖ) - festgestellt werden. Deutlicher erkennbar ist der Trend die menschliche Arbeitswelt aus ökonomischer Perspektive zu betrachten, gleich ob dabei nun stärker das Leistungsprinzip (ÖVP, FPÖ) oder die materielle Existenzsicherung (SPÖ, Grüne) betont werden (Tabelle 4). 
Bei der Ausgestaltung des Wohlfahrtsstaates zeigt sich, ausgenommen bei den Grünen, ein klarer Trend in Richtung der Reduktion von Leistungshöhe und -reichweite. Damit einhergehend wird von den Menschen die Pflicht zur eigenverantwortlichen Vorsorge gefordert (Tabelle 5).

Neoliberale Standpunkte spiegelten sich in allen neuen Schriften wider. Parteibezogen traten die größten Veränderungen zwischen den beiden Grundsatzprogrammen bei der SPÖ hervor. Die aktuellen Programme von ÖVP und FPÖ sind am stärksten von neoliberalen Inhalten gekennzeichnet. Obgleich auch bei den Grünen ein Einstellungswandel identifiziert werden konnte (v. a. in den ersten beiden Dimensionen), ist die Partei in ihren Überzeugungen am wenigsten durch den neoliberalen Zeitgeist beeinflusst.

\section{Literaturverzeichnis}

Altvater, E./Mahnkopf, B. (2007): Grenzen der Globalisierung. Ökonomie, Ökologie und Politik in der Weltgesellschaft. 7. Aufl. Münster: Westfälisches Dampfboot.

Boas, T.C./Gans-Morse, J. (2009): Neoliberalism: Form New Liberal Philosophy to Anti-Liberal Slogan. Studies in Comparative International Development, 44 (2), 137-161.

Butterwegge, C./Lösch, B./Ptak, R. (2008): Kritik des Neoliberalismus. 2., verbesserte Auflage. Wiesbaden: VS Verlag für Sozialwissenschaften.

Clement, R./Terlau, W./Kiy, M. (2012): Angewandte Makroökonomie. Makroökonomie, Wirtschaftspolitik und nachhaltige Entwicklung mit Fallbeispielen. München: Vahlen.

Cockett, R. (1995): Thinking the Unthinkable: Think Tanks and the Economic Counter-Revolution 1931-1983. London: Harper Collins Publisher.

Crouch, C. (2013): Jenseits des Neoliberalismus. Ein Plädoyer für soziale Gerechtigkeit. Wien: Passagen Verlag.

Deppe, H. U. (2005): Zur sozialen Anatomie des Gesundheitssystems. Neoliberalismus und Gesundheitspolitik in Deutschland. 3. Aufl. Frankfurt am Main: Verlag für akademische Schriften.

Die Grünen (1986): Die Grüne Alternative, Liste Freda Meissner-Blau: Grüne Alternativen, für ein neues Österreich. Offenes Kurzprogramm.

Die Grünen (2001): Grundsatzprogramm der Grünen. Beschlossen beim 20. Bundeskongress der Grünen am 07 . und 08. Juli 2001 in Linz.

Dobusch, L./Kapeller, J. (2009): Why is Economics not an Evolutionary Science? New Answers to Veblen's old Question. Journal of Economic Issues, 43 (4), 867-898.

Eucken, W. (1951): Die Entwicklung des ökonomischen Denkens. In: Eucken, W. (Hg.): Unser Zeitalter der Mißerfol- ge. Fünf Vorträge zur Wirtschaftspolitik. Tübingen: Mohr Siebeck, 59-72.

Eucken, W. (1952 [1990]): Grundsätze der Wirtschaftspolitik. 6. Auflage. Tübingen: Mohr Siebeck.

Flechtheim, O. K. (1973): Die Parteien der Bundesrepublik Deutschland. Hamburg: Hoffmann \& Campe.

Flohr, H. (1968): Parteiprogramme in der Demokratie. Ein Beitrag zur Theorie der rationalen Politik. Göttingen: Otto Schwartz.

Freiheitliche Partei Österreich (1973): Freiheitliches Manifest zur Gesellschaftspolitik. Beschlossen von der Bundesparteileitung am 05. und 06. September 1973 in Linz.

Freiheitliche Partei Österreich (2005): Das Parteiprogramm der Freiheitlichen Partei Österreichs. Mit Berücksichtigung der beschlossenen Änderungen vom 27 . Ordentlichen Bundesparteitag der FPÖ am 23.4. 2005 in Salzburg.

Fukuyama, F. (1992): The End Of History and the Last Man. New York: Macmillan.

Gellner, W. (1995): Ideenagenturen für Politik und Öffentlichkeit. Think Tanks in den USA und in Deutschland. Opladen: Westdeutscher Verlag.

Harvey, D. (2005): A Brief History of Neoliberalism. Oxford: Oxford University Press.

Hayek, F. A. (1949 [1960]): The Intellectuals and Socialism. In: Huszar, G. B. de (Hg.): The Intellectuals. A Controversial Portrait. Glencoe, Illinois: The Free Press, 371-384.

Hayek, F. A. (1969): Der Wettbewerb als Entdeckungsverfahren. In: Hayek, F. A. (Hg.): Freiburger Studien. Gesammelte Aufsätze. Tübingen: Mohr Siebeck, 249-265.

Hayek, F. A. (1979 [1996]): Wissenschaft und Sozialismus, In: Hayek, F. A (Hg.): Die Anmaßung von Wissen. Neue Freiburger Studien. Tübingen: Mohr Siebeck, 267-277.

Hayek, F. A. (1980): Recht, Gesetzgebung und Freiheit. Eine neue Darstellung der liberalen Prinzipien der Gerechtigkeit und der politischen Ökonomie. Bd. 1: Regeln und Ordnung. München: Verlag Moderne Industrie.

Heuser, U. J. (2003): Neoliberalismus. Ein Gespenst geht um in Deutschland. Merkur, 57 (9-10), 80o-806.

Hira, A. (1998): Ideas and Economic Policy in Latin America. Regional, National and Organizational Case Studies. London: Praeger.

Kaack, H. (1971): Geschichte und Struktur des deutschen Parteiensystems. Opladen: Westdeutscher Verlag.

Kapeller, J./Huber, J. (2009): Politische Paradigmata und neoliberale Einflüsse am Beispiel von vier sozialdemokratischen Parteien in Europa. Österreichische Zeitschrift für Politikwissenschaft, 38 (2), 163-192.

Keynes, J. M. (1936 [1964]): The General Theory of Employment, Interest and Money. New York: Harcourt Brace \& World.

Klages, J./Strutynski, P. (1997, Hg.): Kapitalismus am Ende des 2o. Jahrhunderts. Hamburg: VSA-Verlag.

Kowall, N. (2013): Das neoliberale Modell. Genese, Politiken, Bilanz. Eine polit- und makroökonomische Bestandsaufnahme. Materialien zu Wirtschaft und Gesell- 
schaft, Nr. 124. Kammer für Arbeiter und Angestellte für Wien. Online: https://emedien.arbeiterkammer.at/ viewer/!fulltext/AC11206616/2/ [01.08.2018].

Kriechbaumer, R. (2006): Die Ära Kreisky. Österreich 19701983. Wien/Köln/Graz: Böhlau Verlag.

Lacina, F. (1993): Austro-Keynesianismus. In: Weber, F./Venus, T. (Hg.): Austro-Keynesianismus in Theorie und Praxis. Wien: Jugend \& Volk Wissenschaft, 15-20.

Marx, K./Engels, F. (1848): Manifest der Kommunistischen Partei. Office der „Bildungs-Gesellschaft für Arbeiter“. London.

Mayring, P. (2010): Qualitative Inhaltsanalyse. Grundlagen und Technik. Weinheim: Beltz Verlag.

Mirowski, P. (2013): Never Let a Serious Crisis go to Waste. How Neoliberalism Survived the Financial Meltdown. New York/London: Verso.

Mirowski, P. (2014): The Political Movement that Dared not Speak its own Name. The Neoliberal Thought Collective Under Erasure. Institute for New Economic Thinking Discussion Paper 14/23, New York.

Mirowski, P./Plehwe, D. (Hg.): The Road from Mont Pelerin. The Making of the Neoliberal Thought Collective. Cambridge, MA/London: Harvard University Press, 2009.

Mont Pèlerin Society (2018): Statement of Aims. Online: https://www.montpelerin.org/statement-of-aims/ [18.01.2018].

Nachtwey, O. (2009): Marktsozialdemokratie. Die Transformation von SPD und Labour Party. Wiesbaden: VS Verlag für Sozialwissenschaften.

Nash, G. H. (1976): The Conservative Intellectual Movement in America. Since 1945. New York: Basic Books.

Nordmann, J. (2005): Der lange Marsch zum Neoliberalismus. Vom Roten Wien zum freien Markt - Popper und Hayek im Diskurs. Hamburg: VSA.

Österreichische Volkspartei (1972): Salzburger Programm. Das Grundsatzprogramm der ÖVP. Beschlossen am 01.12.1972 auf dem außerordentlichen Bundesparteitag in Salzburg.

Österreichische Volkspartei (2015): Grundsatzprogramm 2015 der Österreichischen Volkspartei. Beschlossen am 12.05.2015 im Rahmen des 37. außerordentlichen Bundesparteitag in Wien.

Österreichische Volkspartei (2018): Die Geschichte der Volkspartei. Online: https://www.oevp.at/Die-Geschichte [12.02.2018].

Ötsch, W. O./Pühringer S./Hirte K. (2018): Netzwerke des Marktes. Ordoliberalismus als Politische Ökonomie. Wiesbaden: Springer.

Ötsch, W. O. (2009): Mythos Markt. Marktradikale Propaganda und ökonomische Theorie. Marburg: Metropolis.

Plehwe, D./Wapen, B. (1998): Marktradikale Think Tanks und Stiftungen. International organisierte und institutionalisierte Diskursagglomeration als Kräfteaggregat der neuen Rechten. Forum Wissenschaft, 15 (1), VI-IX.
Plehwe, D./Walpen, B. (1999): Wissenschaftliche und wissenschaftspolitische Produktionsweisen im Neoliberalismus. Beiträge der Mont Pèlerin Society und marktradikaler Think Tanks zur Hegemoniegewinnung und -erhaltung. PROKLA, 29 (2), 203-235.

Ptak, R. (2004): Vom Ordoliberalismus zur Sozialen Marktwirtschaft. Stationen des Neoliberalismus in Deutschland. Opladen: Leske \& Budrich.

Rieger, P. (1993): Die Rezeption keynesianischer Ideen in Österreich. Erinnerungen eines Zeitzeugen. In: Weber, F./ Venus, T. (Hg.): Austro-Keynesianismus in Theorie und Praxis. Wien: Wien Jugend \& Volk Wissenschaft, 29-36.

Saad-Filho, A./Johnston, D. (2005): Neoliberalism. A Critical Reader. London/Ann Arbor: Pluto Press.

Schmelzer, M. (2010): Freiheit für Wechselkurse und Kapital. Die Ursprünge neoliberaler Wirtschaftspolitik und die Mont Pèlerin Society. Marburg: Metropolis.

Smith, A. (1776 [2001]): Der Wohlstand der Nationen. Eine Untersuchung seiner Natur und seiner Ursachen. München: dtv Verlagsgesellschaft.

Sozialistische Partei Österreich (1978): Sozialistische Politik 8. Das neue Programm der SPÖ. Beschlossen vom Bundesparteitag der SPÖ am 20.05.1978.

Sozialdemokratische Partei Österreich (1998): SPÖ-Grundsatzprogramm. Das Programm für eine erfolgreiche $\mathrm{Zu}$ kunft. Beschlossen am 30./31.Oktober 1998.

van Treeck, T./Hein, E./Dünhaupt, P. (2007): Finanzsystem und wirtschaftliche Entwicklung in den USA und in Deutschland im Vergleich. Eine makroökonomische Skizze. WSI Mitteilungen, 6o (12), 635-642.

Walpen, B. (2000): Von Igeln und Hasen oder: Ein Blick auf den Neoliberalismus. UTOPIE kreativ, 121/122, 10661079.

Williamson, J. (1993): Democracy and the „Washington Consensus“. World Development, 21 (8), 1329-1336.

Willke, G. (2003): Neoliberalismus. Frankfurt am Main: Campus Verlag. 